\title{
A comparison of the isolation rates of Salmonella and thermophilic Campylobacter species after direct inoculation of media with a dilute faecal suspension and undiluted faecal material
}

\author{
K. J. NYE, T. TURnER, D. J. COLEMAN, D. FALLON, B. GEE, S. MESSER, R. E. WARREN and \\ N. ANDREWS* on behalf of the PHLS (Midlands) Bacterial Methods Evaluation Group
}

Public Health Laboratory Service (Midlands), Group Headquarters, Royal Shrewsbury Hospital, Mytton Oak Road, Shrewsbury SY3 8XQ and *PHLS Statistics Unit, 61 Colindale Avenue, London NW9 5EQ

\begin{abstract}
Regardless of media used, dilution of faecal samples before direct plating may improve isolation rates and reduce subcultures by freeing organisms from the faecal mass and diminishing competing flora. Despite the routine use of dilution in many laboratories, it has never been established properly whether direct or dilute inocula should be used in primary plating of faeces. A total of 3764 faecal samples was examined in four laboratories with a standardised methodology. The isolation rates, competing flora and confirmatory work performed for Salmonella spp. and Campylobacter spp. from primary plating media with a dilute faecal inoculum were compared with those after direct inoculation of faecal material. Inoculum effects on the isolation of Shigella spp. could not be assessed as only one isolate occurred during the study period. The overall isolation rates of both major enteric pathogens were unaffected by the inoculum. However, significantly fewer wasted subcultures were recorded with a dilute inoculum for Campylobacter spp., and competing flora was reduced in all cases without diluting out small numbers of the pathogen.
\end{abstract}

\section{Introduction}

Several authors [1-3] have identified the requirement for a standardised faecal inoculum when using enrichment media for salmonellae, usually a suspension in quarter-strength Ringer's solution. However, whether a similar dilution step is required for the optimal inoculation of primary plating media has never been established. Many laboratories currently use a dilute inoculum because organisms are released from the faecal mass and competing flora are reduced, allowing better colony separation and facilitating identification. Lynton-Moll and Johnson [3] mentioned that dilution gave a particular advantage over direct plating when low numbers of shigellae are present, but gave no supporting data. If isolation rates from primary plating can be improved by this method, clinicians may receive positive results earlier and laboratory costs may be lowered by a reduction in the number of subcultures.

Received 29 Jan. 2000; revised version accepted 29 June 2000.

Corresponding author: Dr K.J. Nye.
The use of direct and dilute faecal inocula for primary plating was evaluated in four laboratories with a standardised protocol. Data were collected to determine isolation rates of Salmonella spp., Shigella spp. and thermophilic Campylobacter spp., to assess the levels of competing flora present and to gauge the relative amounts of confirmatory work generated.

\section{Materials and methods}

Media

Xylose lysine desoxycholate agar (XLD) (CM469; Oxoid) was prepared according to the manufacturer's recommendations. Campylobacter selective agar (CAMP) (CM739; Oxoid) was used as a base, and supplemented with cefaperazone $32 \mathrm{mg} / \mathrm{L}$ and amphotericin $10 \mathrm{mg} / \mathrm{L}$ (SR155H; Oxoid). Both media were produced at two sites from the same batches to avoid inter-laboratory variations. All media were prepared according to standard protocols and subjected to full quality control procedures before distribution. All plates were used within 14 days of preparation. 


\section{Samples}

Routine faecal samples, received from both hospital and external sources, were included early in the study. Samples that were insufficient for duplicate inoculation, and those submitted for limited examination such as salmonella screening, were excluded. The appearance of samples was recorded as either 'formed' or 'liquid'. For formed samples, a pea-sized portion of faecal material (c. $1 \mathrm{~g}$ ) was emulsified in $3 \mathrm{ml}$ of maximal recovery diluent (MRD) (CM733; Oxoid), i.e., a 1 in 4 dilution. For liquid samples, a 1 in $4 \mathrm{v} / \mathrm{v}$ dilution was made in MRD.

\section{Media inoculation and incubation}

Direct inoculum. A single, sterile, cotton swab was coated in faecal material and a portion was inoculated on to a third of the total surface area of each plate. The inoculum was streaked, with a new sterile loop, to give individual colonies.

Dilute inoculum. A 3-ml pastette was used to inoculate one drop $(c .45 \mu \mathrm{l})$ of faecal suspension on to XLD and two drops on to CAMP, and streaked for single colonies as before.

XLD plates were incubated in air at $36^{\circ} \mathrm{C} \pm 1{ }^{\circ} \mathrm{C}$ for 16-24 h. CAMP plates were incubated micro-aerophilically at $36^{\circ} \mathrm{C} \pm 1{ }^{\circ} \mathrm{C}$ for $40-48 \mathrm{~h}$.

\section{Plate reading}

Plates inoculated directly and with diluted faecal suspension were examined independently by different members of staff. One example of each morphological type of suspect colony was subcultured per plate. Data on bacterial growth and potential pathogens subcultured were recorded at the time of reading and verified daily by a separate, senior member staff. Bacterial growth was recorded semi-quantitatively: 1-10 colonies, light growth $(+) ; 11-50$ colonies, moderate growth $(++)$; and $>50$ colonies, heavy growth $(+++)$. An assessment of competing flora was also made on this basis.

\section{Identification}

Suspected Campylobacter spp., with the typical morph- ology of flat, translucent grey colonies, were confirmed by their appearance on Gram's staining as S-shaped gram-negative bacilli and a positive oxidase test. Possible salmonellae, appearing as red colonies with a black centre on XLD, and shigellae appearing as red colonies, were first screened for urease production. Urease-negative isolates were then identified by the API 10S system (bioMérieux). Those confirmed as Salmonella or Shigella spp. were further identified by standard serological testing. All isolates were referred to the Laboratory of Enteric Pathogens, Central Public Health Laboratory, 61 Colindale Avenue, London, for confirmation.

\section{Statistical methods}

To detect small differences between the methods, the proposed sample size was 1000 per laboratory. The final sample size was just below this with an average of 941 samples per laboratory. The data were entered into an Epi Info Version 6.0 database and analysed in Epi Info [4]. Isolation rates were compared between laboratories and by sample type (liquid/formed) by $\chi^{2}$ tests. The methods (dilute/direct) were compared by an exact binomial test on the discrepancies.

\section{Results}

During July-Sept. 1998, 3764 faecal samples were examined by the four laboratories $(792,838,1000$ and 1134, respectively). Only one Shigella sp. was recovered during the study period, rendering statistical analysis for this organism impossible. Results for Salmonella spp. and thermophilic Campylobacter spp. are summarised in Tables 1 and 2 .

For Salmonella spp., no overall difference in isolation rates could be demonstrated between direct and dilute inocula (difference $=0.05 \% ; 95 \% \mathrm{CI},-0.14-0.22 \%$ ). A significant inter-laboratory variation in isolation rates was seen, from $0.8 \%$ to $2.63 \%(\mathrm{p}=0.007)$; however, this was explained by local variation in food-poisoning rates over the study period, from 30.1/100000 population to $95 / 100000$ population in the corresponding laboratories, compared with a regional mean of 55.7/ 100000 (unpublished data, Communicable Diseases Surveillance Centre, Midlands).

Table 1. Effect of inoculum on isolation rates of Salmonella and Campylobacter spp.

\begin{tabular}{|c|c|c|c|c|c|c|}
\hline \multirow[b]{3}{*}{ Micro-organism } & \multicolumn{6}{|c|}{ Number $(\%)$ of cultures positive } \\
\hline & \multicolumn{2}{|c|}{$\begin{array}{c}\text { Overall } \\
(\mathrm{n}=3764)\end{array}$} & \multicolumn{2}{|c|}{$\begin{array}{l}\text { Liquid samples } \\
\quad(\mathrm{n}=1112)\end{array}$} & \multicolumn{2}{|c|}{$\begin{array}{l}\text { Formed samples } \\
\quad(\mathrm{n}=2649)\end{array}$} \\
\hline & Direct & Dilute & Direct & Dilute & Direct & Dilute \\
\hline Salmonella spp. & $59(1.57 \%)$ & $61(1.62 \%)$ & $26(2.3 \%)$ & $31(2.8 \%)$ & $33(1.2 \%)$ & $30(1.1 \%)$ \\
\hline Campylobacter spp. & $262(6.96 \%)$ & $261(6.93 \%)$ & $132(11.9 \%)$ & $130(11.7 \%)$ & $130(4.9 \%)$ & $131(4.9 \%)$ \\
\hline
\end{tabular}


Table 2. Comparison of semi-quantitative growth estimates for Salmonella and Campylobacter spp. by inoculum

\begin{tabular}{|c|c|c|c|c|c|}
\hline & $\begin{array}{l}\text { Growth } \\
\text { estimate: }\end{array}$ & Growth & estimate: & dilute & inoculum \\
\hline Micro-organism & inoculum & 0 & + & ++ & +++ \\
\hline \multirow[t]{4}{*}{ Salmonella spp. } & 0 & 3698 & 3 & 1 & 3 \\
\hline & + & 3 & 2 & 4 & 3 \\
\hline & ++ & 0 & 1 & 8 & 1 \\
\hline & +++ & 2 & 3 & 2 & 30 \\
\hline \multirow[t]{4}{*}{ Campylobacter spp. } & 0 & 3483 & 7 & 6 & 6 \\
\hline & + & 7 & 6 & 8 & 7 \\
\hline & ++ & 6 & 7 & 11 & 11 \\
\hline & +++ & 7 & 14 & 22 & 156 \\
\hline
\end{tabular}

No significant difference in numbers of colonies of salmonellae isolated from positive samples, estimated semi-quantitatively, was found. The direct inocula gave more colonies than dilute inocula for 11 samples, dilute inocula more than direct inocula for $15(\mathrm{p}=0.55)$. Also there was no evidence that low numbers of salmonellae had been lost by the dilution step (Table 2). Liquid samples were more likely to be positive than formed samples $(2.8 \%$ positive for liquid versus $1.1 \%$ for formed, $p=0.002$ ) and this effect was most apparent in laboratory $\mathrm{C}$ where $11.7 \%$ of liquid samples were positive compared with $1.2 \%$ of formed samples.

More competing flora were apparent with the direct inoculum than after dilution; $67.4 \%$ of directly inoculated samples had a heavy growth compared with $59 \%$ of dilute inocula $(\mathrm{p}<0.001)$. For salmonellapositive samples alone there was also a difference $(50 \%$ direct versus $33 \%$ dilute, $\mathrm{p}=0.035$ ). A difference in both the number and positivity rates of confirmatory tests required was seen in only one laboratory. However, in this laboratory, a small set of liquid samples with a high positivity rate was received and, for only the directly inoculated plates, more than one subculture was made with the second subculture never proving to be a Salmonella spp. (Table 3).

For Campylobacter spp., no overall difference in isolation rates was seen between direct and dilute inocula and, again, liquid samples were more frequently positive $(\mathrm{p}<0.001)$. Of 309 subcultures made from plates inoculated directly, $84.8 \%$ were positive, compared with 284 subcultures of which $91.9 \%$ were positive from dilute inocula $(\mathrm{p}=0.007)$.

Significantly fewer competing flora were detected from dilute inocula than direct $(17.4 \%$ had heavy growth from dilute samples versus $26.5 \%$ for direct plating, $\mathrm{p}<0.001$ ). This effect was evident to a lesser extent in campylobacter-positive samples, but the difference was not statistically significant $(10.7 \%$ versus $13.6 \%$, $\mathrm{p}=0.22$ ). Estimated semi-quantitatively, there was some evidence, although not statistically significant, that numbers of colonies of Campylobacter spp. isolated were lower with dilution (direct inocula gave more than dilute inocula on 62 samples, dilute more than direct on $46, \mathrm{p}=0.15$ ) (Table 2). There was no evidence, overall, that low numbers were diluted out.

Examining discrepant results, where Campylobacter spp. were isolated by only one of the methods, competing flora were consistently greater on the plate which failed to isolate the organism, the effect being larger for direct inocula (direct $>$ dilute $=12: 1$ ) than dilute (dilute $>$ direct $=4: 2$ ). For Campylobacter spp. the pattern of discrepancies between direct and dilute inocula significantly differed between laboratories $(p=0.006)$; this remains unexplained. Two laboratories showed marginal evidence in favour of direct inoculation. One laboratory had better results with dilute inocula and the fourth laboratory showed no difference between the two methods.

\section{Discussion}

Lynton-Moll and Johnson [3] found that one drop of an c. $25 \%$ suspension of faeces in Ringer's solution applied to DCA plates by rotary plating gave good colony separation which facilitated the identification of salmonellae and, more particularly, low numbers of shigellae from known positive faecal samples. However, their data were not well documented and dilution

Table 3. Percentage of urease and API tests indicating a salmonella by laboratory and inoculum

\begin{tabular}{|c|c|c|c|c|c|}
\hline \multirow[b]{2}{*}{ Laboratory } & \multirow[b]{2}{*}{ Inoculum } & \multicolumn{2}{|c|}{ Urease tests } & \multicolumn{2}{|c|}{ API tests } \\
\hline & & $\begin{array}{l}\text { performed } \\
\text { (n) }\end{array}$ & $\begin{array}{c}\text { negative } \\
(\%)\end{array}$ & $\begin{array}{l}\text { performed } \\
\text { (n) }\end{array}$ & $\begin{array}{c}\text { salmonella } \\
\text { confirmed }(\%)\end{array}$ \\
\hline \multirow[t]{2}{*}{ A } & Direct & 127 & 20.5 & 56 & 46.4 \\
\hline & Dilute & 114 & 20.2 & 49 & 46.9 \\
\hline \multirow[t]{2}{*}{ B } & Direct & 77 & 11.6 & 24 & 37.5 \\
\hline & Dilute & 81 & 11.1 & 24 & 37.5 \\
\hline \multirow[t]{2}{*}{$\mathrm{C}$} & Direct & 181 & 9.4 & 79 & 21.5 \\
\hline & Dilute & 58 & 36.2 & 33 & 63.6 \\
\hline \multirow[t]{2}{*}{$\mathrm{D}$} & Direct & 94 & 7.4 & 16 & 43.8 \\
\hline & Dilute & 87 & 9.2 & 20 & 40.0 \\
\hline
\end{tabular}


was not thought to be so effective for other media, such as XLD, bismuth sulphite and brilliant green agar. Moreover, their prime objective was to establish a standardised inoculum for salmonella enrichment media.

In the present study, a 1 in 4 dilution of inoculum had no effect on the overall isolation rates for salmonellae, although as dilution appeared to have little effect on numbers of both competing flora and salmonellae, a higher dilution may give better results. However, for Campylobacter spp. a higher dilution would risk the loss of low numbers of organisms. The differing effects for salmonellae and Campylobacter spp. may be due to the relative selectivity of the media used and incubation conditions, i.e., CAMP media is more inhibitory to enteric micro-organisms and is incubated micro-aerophilically. There was no evidence that direct counts of Campylobacter spp. were generally lower than those of salmonellae and, therefore, more susceptible to dilution effects.

Overall, no disadvantages to the use of a dilute inoculum were seen. The principal advantages of a standardised inoculum and a decreased workload due to fewer wasted subcultures for Campylobacter spp. may partially be offset by the increased work of preparing the inoculum. However, as a faecal suspension is pre- ferable for inoculation of enrichment broths $[1,2]$, this should not pose a problem.

In conclusion, this large multi-centre study showed that the use of a dilute faecal inoculum resulted in significantly fewer wasted subcultures for Campylobacter spp., but had no effect on overall isolation rates of Salmonella spp. or Campylobacter spp. Furthermore, dilution provides a standardised inoculum and an appropriate preparation for inoculation of enrichment broth. Therefore, a faecal suspension of c. $25 \%$ in MRD is recommended for direct inoculation of solid media for the isolation of salmonellae and thermophilic Campylobacter spp.

\section{References}

1. Leifson E. New selenite enrichment media for the isolation of typhoid and paratyphoid (salmonella) bacilli. Am J Hyg 1936; 24: $423-432$.

2. Rappaport F, Konforti N, Navon B. A new enrichment medium for certain salmonellae. J Clin Pathol 1956; 9: 261-266.

3. Lynton-Moll CA, Johnson T. Isolation of salmonella using a standardised inoculum and a rotary plating technique. $J$ Clin Pathol 1978; 31: 904-906.

4. Dean AG, Dean JA, Coulombier D et al. Epi Info, version 6: a word processing, database and statistics program for epidemiology on microcomputers. Centers for Disease Control and Prevention, Atlanta, GA, USA, 1994. 